\title{
Performance Characteristics of the Digital Biograph Vision PET/CT System
}

\author{
Joyce van Sluis, Johan de Jong, Jenny Schaar, Walter Noordzij, Paul van Snick, Rudi Dierckx, Ronald Borra, \\ Antoon Willemsen, and Ronald Boellaard \\ Department of Nuclear Medicine and Molecular Imaging, University Medical Center Groningen, University of Groningen, \\ Groningen, The Netherlands
}

This study evaluated the performance of the Biograph Vision digital PET/CT system according to the NEMA NU 2-2012 standard (published by the National Electrical Manufacturers Association [NEMA]) to allow for a reliable, reproducible, and intersystemcomparable performance measurement. Methods: The new digital PET/CT system features silicon photomultiplier-based detectors with 3.2-mm lutetium oxyorthosilicate crystals and full coverage of the scintillator area. The PET components incorporate 8 rings of 38 detector blocks, and each block contains $4 \times 2$ mini blocks. Each mini block consists of a $5 \times 5$ lutetium oxyorthosilicate array of $3.2 \times 3.2 \times 20 \mathrm{~mm}$ crystals coupled to a silicon photomultiplier array of $16 \times 16 \mathrm{~mm}$, resulting in an axial field of view of $26.1 \mathrm{~cm}$. In this study, PET/CT system performance was evaluated for conformation with the NEMA NU 2-2012 standard, with additional measurements described in the new NEMA NU 2-2018 standard. Spatial resolution, sensitivity, count-rate performance, accuracy of attenuation and scatter correction, image quality, coregistration accuracy, and time-of-flight performance were determined. Measurements were directly compared with results from its predecessor, the Biograph mCT Flow, using existing literature. Moreover, feasibility to comply with the European Association of Nuclear Medicine Research Ltd. (EARL) criteria was evaluated, and some illustrative patient PET images were obtained. Results: The Biograph Vision showed a transverse and axial spatial resolution of 3.6 and $3.5 \mathrm{~mm}$, respectively, in full width at half maximum at a $1-\mathrm{cm}$ offset from the center of the field of view (measured with a ${ }^{22} \mathrm{Na} 0.25-\mathrm{mm}$ point source), a NEMA sensitivity of $16.4 \mathrm{kcps} / \mathrm{MBq}$, and a NEMA peak noise-equivalent count-rate of $306 \mathrm{kcps}$ at $32 \mathrm{kBq} / \mathrm{mL}$. Time-of-flight resolution varied from 210 to 215 as count-rate increased up to the peak noise-equivalent count-rate. The overall image contrast seen with the NEMA image quality phantom ranged from $77.2 \%$ to $89.8 \%$. Furthermore, the system was able to comply with the current and future EARL performance criteria. Conclusion: The Biograph Vision outperforms the analog Biograph mCT Flow, and the system is able to meet European harmonizing performance standards.

Key Words: digital detectors; PET/CT; NEMA; performance evaluation

J Nucl Med 2019; 60:1031-1036

DOI: 10.2967/jnumed.118.215418

\footnotetext{
Received May 31, 2018; revision accepted Dec. 10, 2018.

For correspondence or reprints contact: Joyce van Sluis, Department of Nuclear Medicine and Molecular Imaging, University Medical Center Groningen, Hanzeplein 1, 9713GZ, Groningen, The Netherlands.

E-mail: j.van.sluis@umcg.nl

Published online Jan. 10, 2019.

COPYRIGHT (C 2019 by the Society of Nuclear Medicine and Molecular Imaging.
}

$\mathbf{P}$ ET plays a key role in diagnosis and evaluation of medical conditions. Since 1998, when the first hybrid PET/CT system became operational (1), advances in PET technology have been significant. The implementation of fast lutetium oxyorthosilicate crystals (2) allowed for shorter coincidence timing windows and enabled time-of-flight (TOF) imaging (3-5), and the use of an extended axial field of view (FOV) increased volume sensitivity (6).

Evaluation of the physical performance of PET systems using NEMA NU 2-2012 (published by the National Electrical Manufacturers Association [NEMA]) allows for reproducible and accepted comparisons between PET systems (7).

The digital Biograph Vision PET/CT system (Siemens Healthineers) introduces silicon photomultiplier (SiPM)-based detectors with 3.2-mm lutetium oxyorthosilicate crystals and full coverage between the crystal and the SiPMs. The Vision is the third commercially available digital system, with the other two being the Vereos (Philips Healthcare) $(8,9)$ and the Discovery MI (GE Healthcare) (10).

The purpose of this study was to evaluate the performance of the Vision according to both the NEMA NU 2-2012 (7) and the NEMA NU 2-2018 standards (11). Results were compared with data from the analog Biograph mCT Flow system (12). Spatial resolution, sensitivity, scatter fraction, noise-equivalent count-rate (NECR), image quality, and accuracy of attenuation and scatter corrections were evaluated following the NEMA NU-2 2012 protocol. TOF resolution and coregistration accuracy were determined according to the NEMA NU-2 2018 standard. The feasibility of complying with the European Association of Nuclear Medicine Research Ltd. (EARL) criteria was explored, and some first illustrative patient images were obtained.

\section{MATERIALS AND METHODS}

\section{Biograph Vision PET/CT System}

The Vision combines a 128-slice CT scanner with a whole-body lutetium oxyorthosilicate PET system. The system has a $78-\mathrm{cm}$ bore and $227-\mathrm{kg}$ table capacity.

The PET component contains 8 detector rings and 19 detector electronics assembly units to form a ring. Two adjacent detector blocks per detector electronics assembly unit result in 38 blocks per ring. Each detector block contains a $4 \times 2$ arrangement of mini blocks. A mini block consists of a $5 \times 5$ lutetium oxyorthosilicate array of $3.2 \times 3.2 \times 20 \mathrm{~mm}$ crystals coupled to an SiPM array. Each SiPM array is $16 \times 16 \mathrm{~mm}$ and has 16 output channels.

The arrangement of $4 \times 2$ mini blocks, with 2 mini blocks in the axial direction, results in a $32-\mathrm{mm}$ axial FOV for 1 block. This configuration, 
which uses 8 blocks in the axial direction, has a $25.6-\mathrm{cm}$ axial FOV, or $26.1 \mathrm{~cm}$ including the packing spaces between the blocks.

The design of the detector is based on a square array of small crystals whose area is fully covered by SiPM detector elements, exploiting the full potential of SiPMs. The 3.2-mm crystal size allows for a high system spatial resolution, whereas the full coverage optimizes light collection and enables improved timing resolution and signal-to-noise ratio (13).

\section{Measurements}

Performance measurements included spatial resolution, scatter fraction, sensitivity, count-rate performance, image quality, coregistration accuracy, and timing resolution All measurements were conducted according to the NEMA NU 2-2012 and NEMA NU 2-2018 standards. Acquisition and reconstruction protocols, as well as NEMA analysis software, were provided by the manufacturer. All reported metrics conform with the specifications and definitions provided in the NEMA NU 2 standards.

Spatial Resolution. NEMA NU 2-2012 specifies using a ${ }^{18} \mathrm{~F}-\mathrm{FDG}$ point source smaller than $1 \times 1 \times 1 \mathrm{~mm}$. However, for measuring the spatial resolution on the Vision with smaller crystals, a smaller point source could improve test results (14). NEMA NU 2-2018 therefore recommends purchasing a ${ }^{22} \mathrm{Na}$ point source (11). Thus, a $74-\mathrm{kBq}$, 0.25 -mm-diameter spheric ${ }^{22} \mathrm{Na}$ point source (Eckert and Ziegler Isotope Products) was used.

To comply with the NEMA NU 2-2012 standard, measurements were also performed using an ${ }^{18} \mathrm{~F}$-FDG point source. At the start of data acquisition, a point source of $3.7 \mathrm{kBq}$ of ${ }^{18} \mathrm{~F}-\mathrm{FDG}$ with a length of about $0.3 \mathrm{~mm}$ was prepared in a $0.5-\mu \mathrm{L}$ syringe (Hamilton Co.). The activity at the start of acquisition was sufficiently low to keep deadtime losses and randoms below 5\% of total events. Data were acquired at several positions in the FOV (in $x, y$, and $z$ directions of 0,1 , and 3.3 $\mathrm{cm}\left[\approx^{1 / 1} \mathrm{FOV}_{\mathrm{Z}}\right] ; 0,1$, and $13 \mathrm{~cm}\left[\approx 1 / 2 \mathrm{FOV}_{\mathrm{Z}}\right] ; 0,10$, and $3.3 \mathrm{~cm} ; 0,10$, and $13 \mathrm{~cm} ; 0,20$, and $3.3 \mathrm{~cm}$; and 0,20 , and $13 \mathrm{~cm}$, respectively). At least $2 \times 10^{6}$ coincidence counts were acquired in each position.

The obtained sinogram data were Fourier-rebinned and reconstructed by filtered backprojection using only a standard ramp filter into a $880 \times$ $880 \times 307$ matrix with a $0.8 \times 0.8 \times 0.8 \mathrm{~mm}$ voxel size. The data were reconstructed without attenuation and scatter correction. The spatial resolution was determined according to NEMA NU 2-2012 as the full width at half maximum (FWHM) of the point-spread function (PSF) (7).

Scatter Fraction, Count Losses, and Randoms Measurement. The phantom used for these measurements was a 70-cm-long polyethylene cylinder (20-cm diameter), with a line source inserted axially into the cylinder $4.5 \mathrm{~cm}$ radially from the center. At the start of data acquisition, the line source was filled with $1.2 \mathrm{GBq}$ of ${ }^{18} \mathrm{~F}-\mathrm{FDG}$ to achieve count-rates beyond the expected peak of the NECR. Data were acquired for over $12 \mathrm{~h}$, resulting in 35 frames, each with a 240 -s acquisition time and an interframe delay of $960 \mathrm{~s}$. To account for randoms, online randoms subtraction was applied using the delayed-coincidence-time-window technique (15). Subsequently, scatter fraction and NECR were determined (7).

Sensitivity. Sensitivity was measured using a 70 -cm-long polyethylene tube (inner diameter, $1 \mathrm{~mm}$; outer diameter, $3 \mathrm{~mm}$ ) filled with $5.07 \mathrm{MBq}$ of ${ }^{18} \mathrm{~F}-\mathrm{FDG}$ (at acquisition start) and placed inside 5 concentric aluminum sleeves of equal length with known diameters (7). Five data sets were acquired associated with each of the 5 sleeves (starting with all 5 aluminum sleeves and finishing with a single sleeve) for $300 \mathrm{~s}$ each. The measurements were conducted at the center of the transaxial FOV and repeated at a $10-\mathrm{cm}$ radial offset. Randoms subtraction was applied using the delayed-coincidence-time-window measurement. Next, the system sensitivity was computed (7).

Accuracy of Count Losses and Randoms Corrections. This evaluation used data acquired from the scatter fraction and count-rate measurements. Data were corrected for dead time, randoms, scatter, and attenuation (7). The parameters of the low-dose CT scan used for attenuation correction were an $\mathrm{x}$-ray tube current of $80 \mathrm{mAs}$, a tube voltage of $120 \mathrm{keV}$, and a spiral pitch factor of 0.8 . Scatter was corrected using the extended single-scatter simulation algorithm (16), which discriminates the scattered annihilation radiation according to its differential TOF.

Subsequently, the corrected data were reconstructed using the standard provided whole-body reconstruction algorithm, that is, an ordinary Poisson ordered-subset expectation maximization (OP-OSEM) 3-dimensional (3D) iterative algorithm (17) with 8 iterations, 5 subsets, and no filtering. An image matrix size of $220 \times 220$ was used. By extrapolating the true rate for low activity concentrations (where count losses and randoms can be neglected), count-rate accuracy was estimated.

Image Quality, Accuracy of Attenuation, and Scatter Corrections. The PET NEMA NU2 image-quality phantom (IQ phantom) (PTW) was used to evaluate image quality. The background activity concentration at the start of data acquisition was $5.7 \mathrm{kBq} / \mathrm{mL}{ }^{18} \mathrm{~F}-\mathrm{FDG}$. The 4 smallest spheres were filled with a sphere-to-background ratio of $8: 1$ for the first set of scans and 4:1 for the second set of scans. The remaining 2 largest spheres were filled with nonradioactive water. The IQ phantom was positioned with all spheres aligned in the axial and transaxial center of the FOV. For simulation of a clinical situation with activity outside the FOV, the cylindric scatter phantom was placed axially next to the IQ phantom (7). The line source inside the scatter phantom was filled with approximately $116 \mathrm{MBq}$ of ${ }^{18} \mathrm{~F}-\mathrm{FDG}$ at the start of both data acquisitions.

Two sequential measurements of $240 \mathrm{~s}$ each were acquired for a single bed position after a low-dose CT scan for attenuation correction. Acquisitions were done to simulate a whole-body scan (emission and transmission) of $100 \mathrm{~cm}$ total axial imaging distance in $30 \mathrm{~min}$ of emission imaging. All data were corrected for random coincidences (smoothed random correction), normalization, decay, dead-time losses, scatter, and attenuation. The data were reconstructed using an OP-OSEM 3D-iterative algorithm with 8 iterations and 5 subsets, applying PSF and TOF into a $440 \times 440$ matrix with a voxel size of $1.6 \times 1.6 \times 1.6 \mathrm{~mm}$. The percentages contrast obtained for hot and cold spheres, the background count variability for each sphere, and the accuracies of attenuation and scatter corrections were evaluated.

Coregistration Accuracy. For measurement of coregistration accuracy, a vial was filled with $59.6 \mathrm{MBq}$ of ${ }^{18} \mathrm{~F}-\mathrm{FDG}$ activity (in $0.1 \mathrm{~mL}$ at data acquisition start) and CT contrast $(240 \mathrm{mg} / \mathrm{mL})$ to a volume of no more than $1.4 \mathrm{~mL}$. In total, $115 \mathrm{~kg}$ in nine $11.5-\mathrm{kg}$ increments (which includes the weight of the $11.5-\mathrm{kg}$ L-fixture) were placed on the patient bed. The foam holders provided by the manufacturer were positioned on the L-fixture at 6 locations, 3 points on each of 2 transaxial planes. In the transverse direction (with the coordinate system origin $x$ and $y=0$ and $0 \mathrm{~cm}$, respectively), the foam holders were placed at nominal locations of 0 and $1 \mathrm{~cm}, 0$ and $20 \mathrm{~cm}$, and 20 and $0 \mathrm{~cm}$ on the $x$ and $y$ axes, respectively. In the axial direction (with the coordinate system origin $z=0$, located at the edge of the PET axial FOV), the foam holders were placed in the center of the PET axial FOV $(z=1 / 2$ PET axial FOV) and at 5 and $100 \mathrm{~cm}$ from the tip of the patient table (11). Per location, a low-dose CT scan was performed first and followed by a 3-min PET scan. The total of 6 measurements was performed to determine the centroid within the PET and CT data sets and, subsequently, to calculate the length of the $3 \mathrm{D}$ vector between the CT centroid and the PET centroid (i.e., the coregistration error) (11).

Timing Resolution. The TOF resolution was calculated using the acquired scatter data used for NECR performance, according to a new proposed method $(11,18)$. The timing resolution was calculated as the FWHM of the time distribution of events, after correction for scatter, randoms, and the position of the line source. 
EARL Performance. EARL performance on the Vision was measured to evaluate its ability to meet current EARL guidelines and foreseen 2019 EARL guidelines (19-22).

Measurements were conducted according to EARL standard operating procedures (23). The images were reconstructed using an OP-OSEM 3Diterative algorithm with 4 iterations and 5 subsets, applying TOF, into an image matrix size of $220 \times 220$, resulting in a voxel size of $3.3 \times 3.3 \times$ $1.6 \mathrm{~mm}$. This reconstruction was repeated with 8 iterations, a $5-\mathrm{mm}$ FWHM gaussian filter, and an image matrix size of $220 \times 220$. Additional reconstructions applied resolution modeling, with and without a 7 -mm FWHM gaussian filter and with matrix sizes of both $220 \times 220$ and $440 \times 440$. Reconstructions were performed with corrections for attenuation, scatter, normalization, decay, and dead time. Moreover, all (nonsmoothed) reconstructed images were filtered using gaussian kernels with a FWHM ranging from 1 to $10 \mathrm{~mm}$ in 1-mm steps to derive the optimal combination of reconstruction methods, settings, and filtering to achieve EARL-compliant performance. The latter procedure will allow the definition of EARL-compliant reconstruction protocols for the Vision.

To provide the reader with insight on a possible activity or scantime reduction that may be applied in clinical settings, EARL decay measurements were performed according to a previously published method (24).
Patient Study. A patient study is included to provide the reader with a first impression on clinical performance. We do not intend to provide a detailed and valid intersystem comparison.

A 67-y-old woman (1.64 m tall and weighing $73.1 \mathrm{~kg})$ diagnosed with parkinsonism was injected with $200 \mathrm{MBq}$ of ${ }^{18} \mathrm{~F}-\mathrm{FDG}$. At 30 min after injection, a brain PET/CT study was first performed on an $\mathrm{mCT}$ system for $15 \mathrm{~min}$ and then repeated on the Vision ( $\sim 5$ min after the completion of the mCT study). Data from the mCT were reconstructed using 3D-TOF OP-OSEM with 6 iterations, 21 subsets, and resolution modeling. No filter was used, and the resulting image size was $400 \times 400$ with a voxel size of $2 \times 2 \times$ $2 \mathrm{~mm}$. Data acquired on the Vision were reconstructed using 3DTOF OP-OSEM with 8 iterations and 5 subsets, with resolution modeling into a $440 \times 440$ image matrix with a voxel size of $1.6 \times 1.6 \times 1.6 \mathrm{~mm}$.

Another patient, a 56-y-old woman (1.54 m tall and weighing $67.3 \mathrm{~kg}$ ) diagnosed with metastasized non-small cell lung carcinoma was injected with $215 \mathrm{MBq}$ of ${ }^{18} \mathrm{~F}$-FDG. At $60 \mathrm{~min}$ after injection, a whole-body PET/CT study was performed on an $\mathrm{mCT}$ system using 3-min PET acquisitions per bed position. Data were reconstructed using 3D-TOF OP-OSEM with 3 iterations, 21 subsets, and resolution modeling. A gaussian filter of $5 \mathrm{~mm}$ was applied to the reconstructed images, and the resulting image size was $400 \times 400$ with

TABLE 1

NEMA NU 2-2012 Measurement Results and Some Additional Results According to NEMA NU 2-2018 Standard Acquired on Vision with Direct Comparison to Published Results from mCT Flow (12)

\begin{tabular}{|c|c|c|c|c|c|c|c|}
\hline \multirow[b]{3}{*}{ Parameter } & \multirow[b]{3}{*}{ Distance $^{*}$} & \multicolumn{4}{|c|}{ Measured (Vision) } & \multicolumn{2}{|c|}{$\begin{array}{l}\text { Published }\left({ }^{18} \mathrm{~F}-\mathrm{FDG} \text {, }\right. \\
\text { mCT Flow) }\end{array}$} \\
\hline & & \multicolumn{2}{|c|}{ FWHM (mm) } & \multicolumn{2}{|c|}{ FWTM (mm) } & \multirow[t]{2}{*}{$\mathrm{FWHM}(\mathrm{mm})$} & \multirow[t]{2}{*}{ FWTM (mm) } \\
\hline & & ${ }^{22} \mathrm{Na}$ & ${ }^{18} \mathrm{~F}-\mathrm{FDG}$ & ${ }^{22} \mathrm{Na}$ & ${ }^{18} \mathrm{~F}-\mathrm{FDG}$ & & \\
\hline \multicolumn{8}{|l|}{ Spatial resolution } \\
\hline \multirow[t]{3}{*}{ Radial } & 1 & 3.5 & 3.7 & 6.8 & 7.4 & 4.3 & 8.6 \\
\hline & 10 & 4.5 & 4.6 & 8.4 & 8.8 & 5.2 & 9.3 \\
\hline & 20 & 5.8 & 6.0 & 10.5 & 11.1 & 5.6 & 9.8 \\
\hline \multirow[t]{3}{*}{ Tangential } & 1 & 3.6 & 3.7 & 6.9 & 7.2 & 4.3 & 8.6 \\
\hline & 10 & 3.9 & 3.9 & 7.0 & 7.3 & 4.7 & 9.7 \\
\hline & 20 & 3.5 & 3.6 & 6.4 & 7.0 & 6.5 & 12.7 \\
\hline \multirow[t]{3}{*}{ Axial } & 1 & 3.5 & 3.8 & 7.1 & 7.6 & 4.3 & 8.6 \\
\hline & 10 & 4.3 & 4.3 & 8.7 & 9.2 & 5.9 & 11.1 \\
\hline & 20 & 4.4 & 4.6 & 9.4 & 10.2 & 7.8 & 13.7 \\
\hline \multirow[t]{2}{*}{ Sensitivity (kcps/MBq) } & 0 & 16.4 & & & & 9.6 & \\
\hline & 10 & 16.3 & & & & 9.6 & \\
\hline \multicolumn{8}{|l|}{ Accuracy (kcps at kBq/mL) } \\
\hline Peak NECR & \multicolumn{3}{|c|}{306 at 32.6} & \multicolumn{4}{|c|}{185 at 29} \\
\hline Peak true rate & \multicolumn{3}{|c|}{1,306 at 54} & \multicolumn{4}{|c|}{634 at 42.4} \\
\hline \multicolumn{8}{|l|}{ Scatter fraction (\%) } \\
\hline At peak NECR & \multicolumn{3}{|c|}{38.7} & \multicolumn{4}{|c|}{33.4} \\
\hline At low activity & \multicolumn{3}{|c|}{37} & \multicolumn{4}{|c|}{33.5} \\
\hline TOF resolution (ps) & \multicolumn{3}{|c|}{210} & \multicolumn{4}{|c|}{540} \\
\hline Maximum coregistration error $(\mathrm{mm})$ & \multicolumn{3}{|c|}{1.25} & \multicolumn{4}{|c|}{ NA } \\
\hline $\begin{array}{l}{ }^{*} \text { Radial distance }(\mathrm{cm}) \text { from center } F C \\
\text { FWTM = full width at tenth maximur }\end{array}$ & 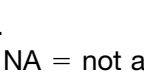 & cable. & & & & & \\
\hline
\end{tabular}


a voxel size of $2 \times 2 \times 2 \mathrm{~mm}$. Subsequently, measurements were repeated on the Vision using 3-min PET acquisitions per bed position. The vendor-recommended reconstruction protocol was applied, that is, 3DTOF OP-OSEM with 4 iterations, 5 subsets, resolution modeling, no filtering, an image matrix size of $220 \times 220$, and a voxel size of $3.3 \times 3.3 \times$ $1.6 \mathrm{~mm}$.

The patient study was approved by the medical ethics review board of the University Medical Center Groningen, and both patients provided written informed consent.

\section{RESULTS}

\section{NEMA Measurements}

Spatial Resolution, Sensitivity, Coregistration Accuracy, and Timing Resolution. The spatial resolutions are summarized in Table 1, listing FWHM and full-width-at-tenth-maximum values at 1,10 , and $20 \mathrm{~cm}$. The sensitivity values for both the 0 - and the $10-\mathrm{cm}$ off-center position, the maximum coregistration error, and the calculated timing resolution are also given in Table 1 . These results show an average sensitivity increase of $70.3 \%$ for the Vision compared with the mCT Flow. The observed TOF of 210 ps worsens only 5 ps from low count-rate up to peak NECR (Table 1; Fig. 1). In addition, the axial sensitivity profiles for both the 0 - and the $10-\mathrm{cm}$ off-center positions are shown in Figure 2.

Scatter Fraction, Count Losses, and Randoms Measurement. Peak NECR and scatter fractions at peak NECR and at low activity levels are given in Table 1. Figure 1 shows plots of the trues, randoms, prompts, and scatter event rates next to the scatter fraction curve as a function of activity. In addition, the NECR as a function of activity concentration is shown.

The trues rate was $1,306 \mathrm{kcps}$ at $54 \mathrm{kBq} / \mathrm{mL}$, and the accuracy mean bias was $2.9 \%$. Figure 3 shows a plot of the maximum and minimum relative count-rate error for the different activity concentrations.

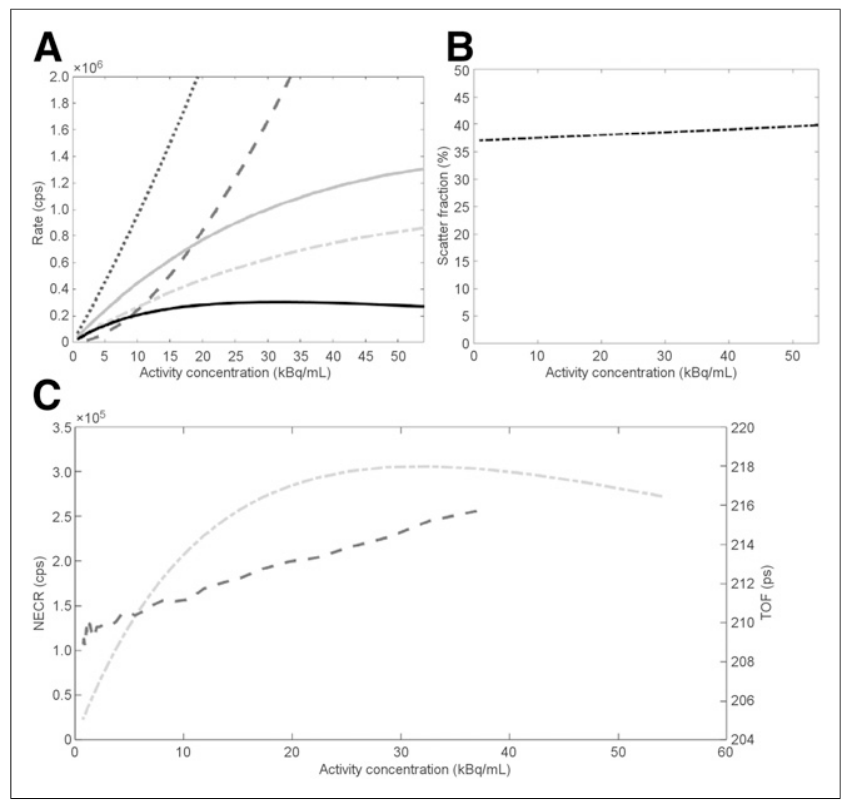

FIGURE 1. (A) Plots of prompts (dotted line), randoms (dashed line), trues (gray solid line), scatter event rates (dashed-dotted line), and NECR (black solid line). (B) Scatter fraction as function of activity concentration. (C) NECR (dashed-dotted line) and TOF (dashed line) as function of activity concentration.

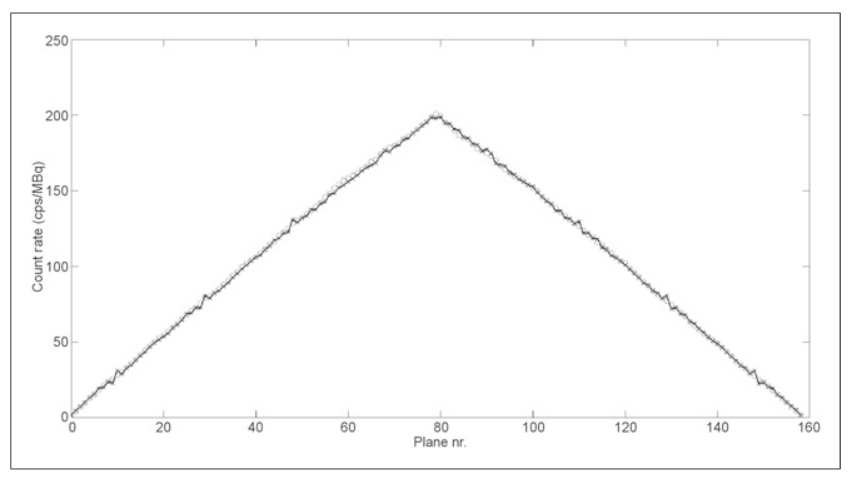

FIGURE 2. Axial sensitivity profiles for both $0-\mathrm{cm}$ off-center position (circles) and 10-cm off-center position (crosses).

Image Quality, Accuracy of Attenuation, and Scatter Corrections. Tables 2 and 3 show the percentage contrast, background variability, and average lung residual for the $8: 1$ and $4: 1$ sphereto-background ratios.

\section{Additional Measurements}

EARL Compliance. Figures 4 and 5 show SUV recovery coefficients as a function of the sphere sizes in the NEMA IQ phantom for various reconstruction protocols according to the current EARL performance criteria $(19,20)$ and foreseen new EARL performance criteria $(21,22)$, respectively.

EARL compliance was achieved by using 3D-TOF OP-OSEM with 4 or 8 iterations and 5 subsets, with a 5-mm FWHM gaussian filter and an image matrix size of $220 \times 220$, or by using 3D-TOF OP-OSEM with resolution modeling and the same reconstruction settings as above but with a 7-mm FWHM gaussian filter and an image matrix size of $220 \times 220$ or $440 \times 440$. For the foreseen new EARL specifications, compliance can be achieved either by using 3D-TOF OP-OSEM with 4 or 8 iterations and 5 subsets, a matrix of $220 \times 220$, and no additional filtering or by using 3D-TOF OPOSEM with resolution modeling, a gaussian filter of 5-mm FWHM, and an image matrix size of either $220 \times 220$ or $440 \times 440$, although at present borderline results were seen using $\mathrm{SUV}_{\text {peak }}$ recoveries. There is no $\mathrm{SUV}_{\text {peak }}$ upper and lower limit according to current EARL specifications; therefore, these limits cannot be shown in Figure 4C.

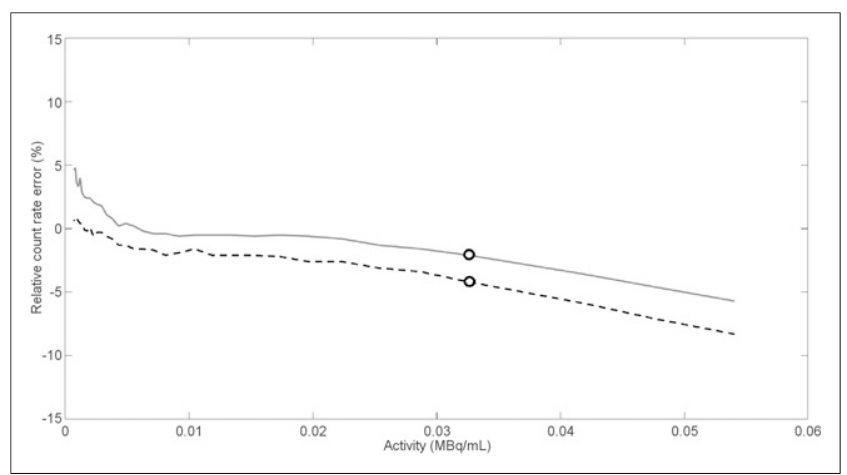

FIGURE 3. Maximum (solid line) and minimum (dashed line) relative count-rate error for different activity concentrations, and maximum and minimum bias values (circles) at activity concentration of peak NECR. 
TABLE 2

Contrast, Background Variability, and Average Lung Residual for 8:1 Sphere-to-Background Ratio on Vision, Directly Compared with Published Results from mCT Flow

\begin{tabular}{|c|c|c|c|c|}
\hline \multirow[b]{2}{*}{ Sphere size $(\mathrm{mm})$} & \multicolumn{2}{|c|}{ Contrast (\%) } & \multicolumn{2}{|c|}{$\begin{array}{l}\text { Background } \\
\text { variability (\%) }\end{array}$} \\
\hline & Vision & mCT Flow & Vision & mCT Flow \\
\hline 10 & 86.8 & 41.9 & 6.0 & 6.3 \\
\hline 13 & 77.2 & 63.1 & 5.0 & 5.4 \\
\hline 17 & 85.0 & 68.1 & 3.9 & 4.4 \\
\hline 22 & 89.8 & 76.6 & 3.3 & 3.6 \\
\hline 28 & 87.4 & 71.3 & 3.0 & 3.0 \\
\hline 37 & 89.6 & 77.7 & 2.2 & 2.4 \\
\hline $\begin{array}{l}\text { Average lung } \\
\text { residual (\%) }\end{array}$ & 3.5 & 12.1 & & \\
\hline
\end{tabular}

mCT Flow measurements include effect of low-resolution matrix and postreconstruction 3-mm gaussian filter (12).

For illustrative purposes, the $\mathrm{SUV}_{\max }$ and $\mathrm{SUV}_{\text {mean }}$ recovery coefficients without filtering, and with and without additional PSF resolution modeling (not EARL-compliant), are shown in Supplemental Figures 1 and 2 (supplemental materials are available at http:// jnm.snmjournals.org). In addition, the results of the EARL decay measurements (24) to provide first insights on possible activity or scan time reduction are shown in Supplemental Tables 1-3; these results suggest that for EARL-compliant reconstructions, a reduction in the activity and scan duration product by a factor of 8 , compared with current recommendations, seems feasible.

TABLE 3

Contrast, Background Variability, and Average Lung Residual for 4:1 Sphere-to-Background Ratio on Vision, Directly Compared with Published Results from mCT Flow

\begin{tabular}{|c|c|c|c|c|}
\hline \multirow[b]{2}{*}{ Sphere size $(\mathrm{mm})$} & \multicolumn{2}{|c|}{ Contrast (\%) } & \multicolumn{2}{|c|}{$\begin{array}{c}\text { Background } \\
\text { variability (\%) }\end{array}$} \\
\hline & Vision & mCT Flow & Vision & mCT Flow \\
\hline 10 & 93.1 & 28.3 & 6.4 & 6.9 \\
\hline 13 & 73.5 & 47.9 & 5.0 & 6.8 \\
\hline 17 & 79.0 & 58.4 & 4.0 & 5.6 \\
\hline 22 & 87.0 & 70.8 & 3.1 & 4.7 \\
\hline 28 & 86.3 & 67.0 & 2.7 & 3.8 \\
\hline 37 & 89.4 & 76.9 & 2.2 & 2.9 \\
\hline $\begin{array}{l}\text { Average lung } \\
\text { residual (\%) }\end{array}$ & 3.4 & 12.3 & & \\
\hline
\end{tabular}

mCT Flow measurements include effect of low-resolution matrix and postreconstruction 3-mm gaussian filter (12).

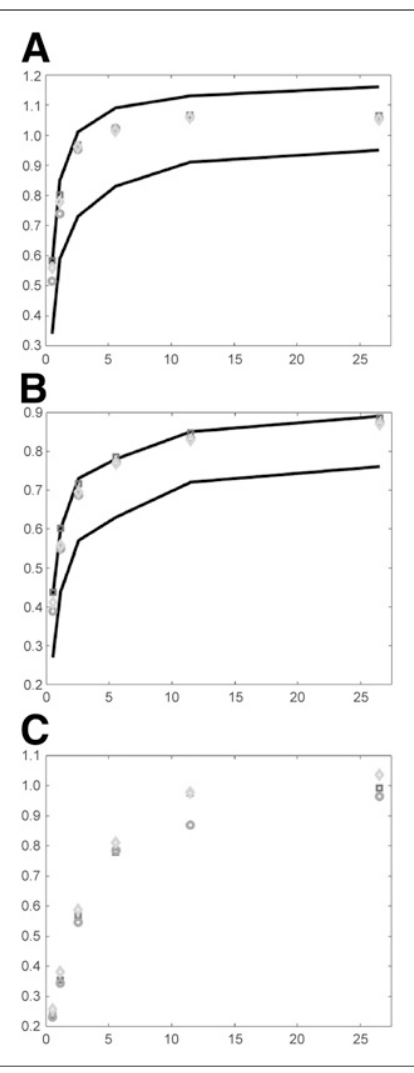

FIGURE 4. SUV recovery coefficients as function of sphere size in $\mathrm{IQ}$ phantom for various reconstruction protocols. Square $=3 \mathrm{D}-\mathrm{TOF}$ OP-OSEM with 4 iterations, 5 subsets, and 5-mm gaussian filter into matrix size of $220 \times 220$; circle $=$ "square" but with 7-mm gaussian filter and addition of PSF; diamond = "circle" but with image size of $440 \times$ 440. According to EARL specifications, $\operatorname{SUV}_{\max }(A), \mathrm{SUV}_{\text {mean }}(\mathrm{B})$, and SUV $_{\text {peak }}(\mathrm{C})$ recoveries are shown. Lines illustrate upper and lower limits. SUV $_{\text {peak }}$ limits are not provided here since these are not included in current EARL guidelines.

Sensitivity and Timing Resolution. The improved TOF resolution of 210 ps can be translated to more effective noise reduction or better contrast enhancement in comparison to the mCT Flow (27).

The higher sensitivity of the Vision may allow for reduction in dose or scan time in future clinical application (Supplemental Tables 1-3 provide first insights regarding dose or scan-time reduction).

Scatter Fraction, Count Losses, and Randoms Measurement. The peak NECR increased $65 \%$ when measured on the Vision, compared with the mCT Flow. Because of the extended axial FOV of the new system and a greater acceptance angle, a small increase in scatter fraction can be expected. The true counts captured on the Vision have increased with respect to its predecessor ( $\sim 770 \mathrm{kcps}$ at $20 \mathrm{kBq} / \mathrm{mL}$ for the Vision and 440 at $20 \mathrm{kBq} / \mathrm{mL}$ for the mCT Flow). The increase in true count-rate is assumed to result from a lower dead time and a higher sensitivity on the Vision than on the mCT Flow; 


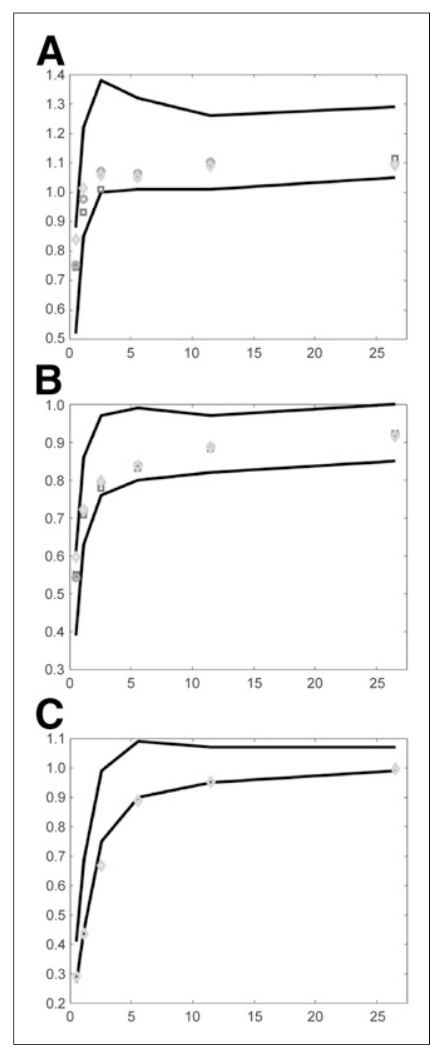

FIGURE 5. SUV recovery coefficients as function of sphere sizes in NEMA NU 2 IQ phantom for various reconstruction protocols. Square $=$ 3D-TOF OP-OSEM with 4 iterations, 5 subsets, and $4-\mathrm{mm}$ gaussian filter into matrix size of $220 \times 220$; circle $=$ "square" but with 5 -mm gaussian filter and addition of PSF; diamond = "circle" but with image size of $440 \times$ 440. According to EARL 2019 specifications, SUV $\max (A), S U V_{\text {mean }}(B)$, and $\mathrm{SUV}_{\text {peak }}(\mathrm{C})$ recoveries are shown. Lines illustrate upper and lower limits.

\section{CONCLUSION}

The Vision had a transverse and axial spatial resolution of 3.6 and $3.5 \mathrm{~mm}$, respectively, at a $1-\mathrm{cm}$ offset from the center of the FOV (measured with a ${ }^{22} \mathrm{Na}$ source), compared with respective values of 4.3 and $4.3 \mathrm{~mm}$ for the mCT Flow. Moreover, compared with the mCT Flow, the Vision had a $70.3 \%$ increase in sensitivity, a $65 \%$ higher peak NECR, and a higher contrast recovery. Finally, the timing resolution improved from $540 \mathrm{ps}$ on the mCT Flow to $210 \mathrm{ps}$ on the Vision. The Vision outperformed the analog mCT Flow in every NEMA performance test that was evaluated.

\section{DISCLOSURE}

The research presented in this study is financially supported by Siemens Molecular Imaging under a collaborative research contract. No other potential conflict of interest relevant to this article was reported.

\section{REFERENCES}

1. Beyer T, Townsend DW, Brun T, et al. A combined PET/CT scanner for clinical oncology. J Nucl Med. 2000;41:1369-1379.

2. Melcher CL. Scintillation crystals for PET. J Nucl Med. 2000;41:1051-1055.

3. Moses WW. Time of flight in PET revisited. IEEE Trans Nucl Sci. 2003;50:1325-1330.

4. Surti S, Kuhn A, Werner ME, Perkins AE, Kolthammer J, Karp JS. Performance of Philips Gemini TF PET/CT scanner with special consideration for its time-offlight imaging capabilities. J Nucl Med. 2007;48:471-480.

5. Jakoby BW, Bercier Y, Conti M, Casey ME, Bendriem B, Townsend DW. Physical and clinical performance of the mCT time-of-flight PET/CT scanner. Phys Med Biol. 2011;56:2375-2389.

6. Jakoby BW, Bercier Y, Watson CC, Bendriem B, Townsend DW. Performance characteristics of a new LSO PET/CT scanner with extended axial field-of-view and PSF reconstruction. IEEE Trans Nucl Sci. 2009;56:633-639.

7. National Electrical Manufacturers Association Performance Measurements of Positron Emission Tomographs. Rosslyn, VA: National Electrical Manufacturers Association; 2012. NEMA Standards Publication NU 2-2012.

8. Nguyen NC, Vercher-Conejero JL, Sattar A, et al. Image quality and diagnostic performance of a digital PET prototype in patients with oncologic diseases: initial experience and comparison with analog PET. J Nucl Med. 2015;56:1378-1385.

9. Rausch I, Ruiz A, Valverde-Pascual I, Cal-Gonzalez J, Beyer T, Carrio I. Performance evaluation of the Philips Vereos PET/CT system according to the NEMA NU2-2012 standard. $J$ Nucl Med. October 25, 2018 [Epub ahead of print].

10. Hsu DFC, Ilan E, Peterson WT, Uribe J, Lubberink M, Levin CS. Studies of a next-generation silicon-photomultiplier-based time-of-flight PET/CT system. J Nucl Med. 2017;58:1511-1518.

11. National Electrical Manufacturers Association Performance Measurements of Positron Emission Tomographs. Rosslyn, VA: National Electrical Manufacturers Association; 2018. NEMA Standards Publication NU 2-2018.

12. Rausch I, Cal-González J, Dapra D, et al. Performance evaluation of the Biograph mCT Flow PET/CT system according to the NEMA NU2-2012 standard. EJNMMI Phys. 2015;2:26.

13. Surti S. Update on time-of-flight PET imaging. J Nucl Med. 2015;56:98-105.

14. St. James ST, Thompson CJ. Investigation of the block effect in LSO detectors. IEEE Nucl Sci Symp Conf Rec. 2005;5:2474-2477.

15. Watson CC, Casey ME, Eriksson L, Mulnix T, Adams D, Bendriem B. NEMA NU 2 performance tests for scanners with intrinsic radioactivity. $\mathrm{J} \mathrm{Nucl} \mathrm{Med}$. 2004;45:822-826.

16. Watson CC. Extension of single scatter simulation to scatter correction of timeof-flight PET. IEEE Trans Nucl Sci. 2007;54:1679-1686.

17. Varrone A, Sjöholm N, Eriksson L, Gulyás B, Halldin C, Farde L. Advancement in PET quantification using 3D-OP-OSEM point spread function reconstruction with the HRRT. Eur J Nucl Med Mol Imaging. 2009;36:1639-1650.

18. Wang G-C, Li X, Niu X, et al. PET timing performance measurement method using NEMA NEC phantom. IEEE Trans Nucl Sci. 2016;63:1335-1342.

19. Boellaard R, Doherty MJO, Weber WA, et al. FDG PET and PET/CT: EANM procedure guidelines for tumour PET imaging: version 1.0. Eur J Nucl Med Mol Imaging. 2010;37:181-200.

20. Boellaard R, Delgado-Bolton R, Oyen WJG, et al. FDG PET/CT: EANM procedure guidelines for tumour imaging: version 2.0. Eur J Nucl Med Mol Imaging. 2015;42:328-354.

21. Kaalep A, Sera T, Rijnsdorp S, et al. Feasibility of state of the art PET/CT systems performance harmonisation. Eur J Nucl Med Mol Imaging. 2018;45:1344-1361.

22. Boellaard R, Sera T, Kaalep A. New EARL PET/CT performance standards for oncological PET/CT studies. EARL website. http://earl.eanm.org/html/img/pool/ New_EARL_project_and_update_of_FDG_standard_Oct2018.pdf. Accessed February 26, 2019.

23. Manual for EARL FDG-PET/CT Accreditation: Version 1.2. Vienna, Austria: EANM Research Ltd.; March 2017.

24. Boellaard R, Willemsen AT, Arends B, Visser EP. EARL procedure for assessing PET/CT system specific patient FDG activity preparations for quantitative FDG PET/CT studies. EARL website. http://earl.eanm.org/html/img/pool/ EARL-procedure-for-optimizing-FDG-activity-for-quantitative-FDG-PETstudies_version_1_1.pdf. Published April 2013. Accessed February 26, 2019.

25. Vandenberghe S, Daube-Witherspoon ME, Lewitt RM, Karp JS. Fast reconstruction of 3D time-of-flight PET data by axial rebinning and transverse mashing. Phys Med Biol. 2006;51:1603-1621.

26. Jødal L, Le Loirec C, Champion C. Positron range in PET imaging: non-conventional isotopes. Phys Med Biol. 2014;59:7419-7434.

27. Karp JS, Surti S, Daube-Witherspoon ME, Muehllehner G. The benefit of timeof-flight in PET imaging: experimental and clinical results. J Nucl Med. 2008;49: $462-470$. 